\title{
Brown's syndrome: an unusual ocular complication of rheumatoid arthritis
}

\author{
C Cooper, J R Kirwan, N W McGill, P A Dieppe
}

\begin{abstract}
A 55 year old man with rheumatoid vasculitis and an apparent left inferior oblique palsy is described. This unusual extraocular complication of rheumatoid arthritis probably resulted from a tenosynovitis of the superior oblique tendon sheath, and resolved with steroid treatment.
\end{abstract}

Ophthalmic disease is a common manifestation of rheumatoid vasculitis. Although this usually presents as scleritis or corneal destruction, other ocular features are recognised. ${ }^{1}$ We report here a patient with rheumatoid vasculitis who concomitantly developed an isolated, unilateral, apparent palsy of his left inferior oblique muscle. The pathogenesis and management of this unusual ophthalmic manifestation of rheumatoid arthritis are discussed.

\section{Case report}

A 55 year old man with a 23 year history of rheumatoid factor positive, nodular, erosive rheumatoid arthritis was admitted with weight loss, malaise, and a flare of his disease. He had previously been treated with gold and was currently being treated with penicillamine. Physical examination showed widespread rheumatoid deformities with moderately active synovitis. There was evidence, however, of the recent onset of cutaneous vasculitis and basal crepitations in both lung fields. Investigations showed haemoglobin $129 \mathrm{~g} / \mathrm{l}$, white cell count $10.4 \times 10^{9} / 1$, normal film and platelets, normal urea, electrolytes, and liver function tests. C reactive protein was $95 \mathrm{mg} / \mathrm{l}($ normal $<10)$ and he was seropositive for rheumatoid factor (titre $1 / 80$ ). His factor VIII related antigen level was 2.86 (normal $<2 \cdot 0$ ) and tests for circulating immune complexes $(\mathrm{C} 3, \mathrm{C} 4$, anticomplementary activity, and $\mathrm{Clq}$ binding) were positive. Radiographs showed erosive rheumatoid arthritis with atlantoaxial subluxation and diffuse lower zone pulmonary shadowing. His carbon monoxide transfer factor was reduced.

During the course of the admission he experienced the sudden onset of painful diplopia. This was maximal on right upward gaze, and ophthalmological examination showed an apparently isolated left inferior oblique palsy (figure). There was associated pain in the left eye, exacerbated by movement upward and inward.

He was initially thought to have a microvascular complication of rheumatoid arthritis and was treated with methylprednisolone $1 \mathrm{~g}$ intravenously. His vasculitis and his ocular condition settled rapidly.

Two weeks later he was readmitted as an emergency with recurrence of a painful apparently isolated left inferior oblique palsy. At this
Rheumatology Unit, University Department of Medicine, Bristol Royal Infirmary, Bristol BS2 8HW C Cooper

J R Kirwan N W McGill P A Dieppe

Correspondence to: Dr Cooper.

Accepted for publication 1 June 1989

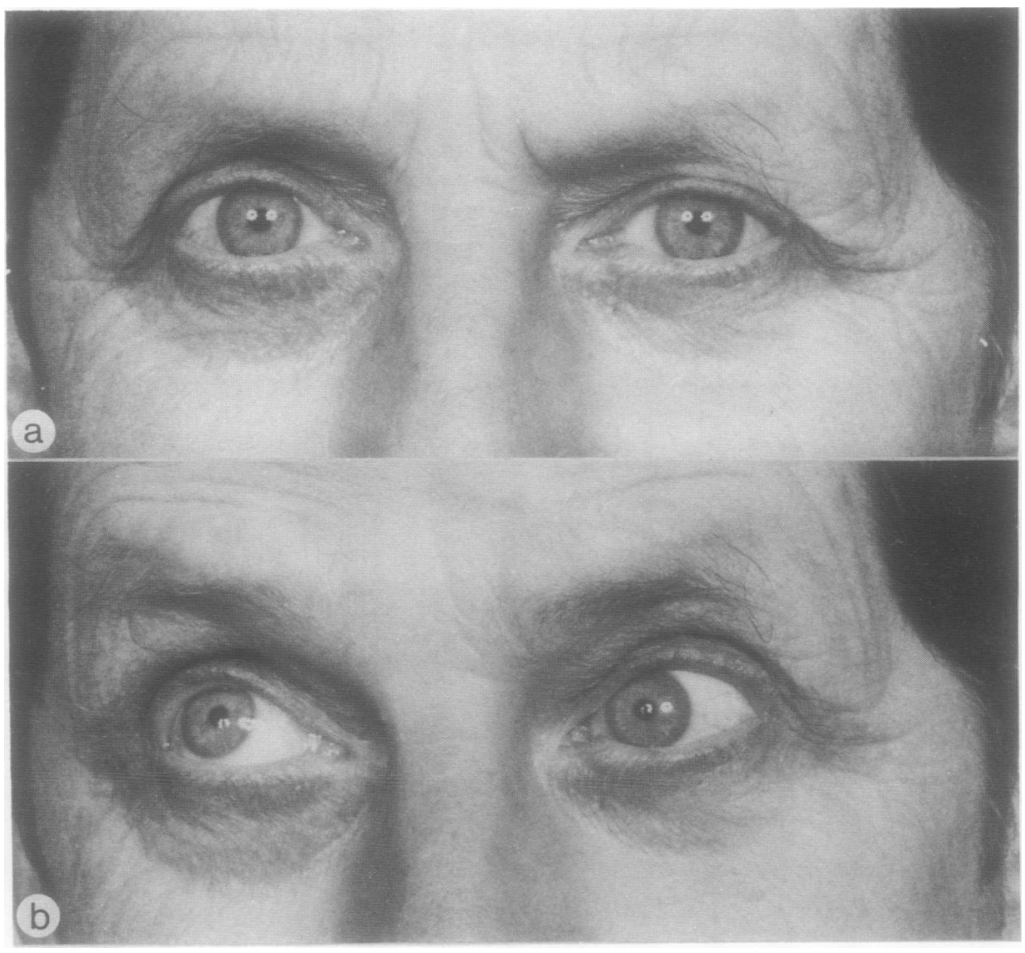

(a) Straight eyes in the primary position;

(b) Brown's syndrome: apparently isolated lefi inferior oblique weaknes resulting in inability to raise the left eye in adduction. 
admission, however, it was noted that continued adduction and elevation of the affected eye resulted in improvement in the diplopia. The presence of a rheumatoid nodule involving the ocular tendons was sought, but a magnetic resonance image of the orbit showed no clear abnormality, and a clinical diagnosis of Brown's syndrome was made.

\section{Discussion}

In 1950 Brown reported the most common cause of an apparently isolated inferior oblique palsy to be an impairment in the compensatory lengthening of its antagonist muscle: the superior oblique. ${ }^{2}$ Normal upward and medial gaze requires a loose superior oblique tendon sheath and free movement of the tendon in both the sheath and the trochlea. Impairment of this movement occurs if the sheath is congenitally shortened (true superior oblique sheath syndrome) or if mechanical obstruction arises from some acquired abnormality. Although this acquired abnormality is rarely identified, cases have been reported in association with trauma and rheumatoid arthritis. ${ }^{3-7}$ In the latter a stenosing tenosynovitis of the superior oblique tendon sheath causes a mechanical obstruction on attempting to raise the eye in adduction. Despite the common association of tenosynovitis at other sites with rheumatoid arthritis this particular complication has been reported only twice. $^{67}$

The pathology of Brown's syndrome in rheumatoid arthritis resembles that of trigger finger. ${ }^{4}$ In both conditions tendons are obstructed in their passage through a fibrous canal by inflammation in their sheaths, and movement resulting from action of the opposing muscle groups is limited. Yet the reported incidence of Brown's syndrome is considerably lower than that of trigger finger.

Sandford Smith reported a woman with longstanding seropositive rheumatoid arthritis who developed an intermittent superior oblique syndrome. ${ }^{4}$ Killian et al have stressed the intermittent nature of the symptoms and a clicking sensation in the region of the trochlea. ${ }^{6}$ Specific treatment is not usually required, though surgical correction has been attempted. ${ }^{6}$

This further case warrants reporting for several reasons. Firstly, the temporal association with vasculitis led to a mistaken initial assumption that the ophthalmoplegia represented a microvascular complication of rheumatoid arthritis. Secondly, the condition in our patient resolved with systemic corticosteroid treatment. Thirdly, despite magnetic resonance imaging it was not possible to show a rheumatoid nodule as the cause of the tendon sheath obstruction. Finally, the case serves to highlight this uncommon ocular manifestation of rheumatoid arthritis.

1 Scott D G I, Bacon P A, Tribe C R. Systemic rheumatoid vasculitis: a clinical and laboratory study of 50 cases. Medicine (Baltimore) 1981; 60: 288-97.

2 Brown H W. Congenital structural muscle anomalies. In: Allen J, ed. Strabismus ophthalmic symposium 1. St Louis: Mosby, 1950: 205.

3 Goldstein J H. Intermittent superior oblique tendon sheath syndrome. Am $\mathcal{F}$ Ophthalmol 1969; 67: 960-2.

4 Sandford-Smith J H. Intermittent superior oblique tendon sheath syndrome: a case report. Br $\mathcal{F}$ Ophthalmol 1969; 53: 412-7.

5 Goldhammer Y, Lawton Smith J. Acquired intermittent Brown's syndrome. Neurology 1974; 24: 666-8

6 Killian P J, McClain B, Lawless O J. Brown's syndrome. An unusual manifestation of rheumatoid arthritis. Arthritis Rheum 1977; 20: 1080-4.

7 Kemp A S, Searle C, Horne S. Transient Brown's syndrome in juvenile chronic arthritis. Ann Rheum Dis 1984; 43: $764-5$. 